\title{
La comportement démographique des groupes linguistiques au Québec pendant la période 1976-1981
}

Une analyse multirégionale

\section{The Demographic Behavior of Linguistic Groups in Quebec} During the 1976-1981 Period

A Multiregional Analysis

\section{El comportamiento demográfico de los grupos linguísticos en Quebec durante el período 1976-1981}

\section{Un analisis multiregional}

\section{Marc Termote et Danielle Gauvreau}

Volume 14, numéro 1, avril 1985

Démolinguistique

URI : https://id.erudit.org/iderudit/600556ar

DOI : https://doi.org/10.7202/600556ar

Aller au sommaire du numéro

Éditeur(s)

Association des démographes du Québec

ISSN

0380-1721 (imprimé)

1705-1495 (numérique)

Découvrir la revue

Citer cet article

Termote, M. \& Gauvreau, D. (1985). La comportement démographique des groupes linguistiques au Québec pendant la période 1976-1981 : une analyse multirégionale. Cahiers québécois de démographie, 14(1), 31-58.

https://doi.org/10.7202/600556ar

\section{Résumé de l'article}

L'objectif de cet article est d'analyser de façon simultanée, selon ce qu'il est convenu d'appeler l'approche multirégionale, les interactions entre le comportement de fécondité, de migration et de mortalité de chacun des principaux groupes linguistiques du Québec. Les résultats de cette analyse montrent que, sur la base du régime démographique observé au cours de la période 1976-1981, la migration a sans doute pour effet de réduire les disparités régionales dans le comportement de fécondité et de mortalité, mais que par contre elle amplifie considérablement l'impact démographique des disparités linguistiques de ce comportement. 
Cahiers québécois de démographie

Vo1. 14, no 1, avril 1985

\title{
Le comportement démographique des groupes linguistiques au Québec pendant la période 1976-1981. Une analyse multirégionale
}

\author{
Marc TERMOTE* et Danielle GAUVREAU**
}

\section{INTRODUCTION}

L'ampleur du phénomène de la mobilité linguistique et géographique relègue souvent au second plan l'analyse des disparités linguistiques en matière de fécondité et de mortalité, surtout dans sa dimension régionale. Et même si l'on connaît relativement bien les différences de comportement migratoire selon les groupes linguistiques, le plus souvent $1^{\prime}$ on se contente d'analyser la migration séparément de la fécondité et de la mortalité, c'est-à-dire qu'on néglige le fait qu'un flux de migration représente pour la région de départ non seulement la perte d'un certain nombre d'individus, mais également la perte d'un certain nombre d'kannées à vivrell et d'un certain nombre d' "enfants à naître".

Or, depuis 1975, l'enregistrement des événements d'état civil fait l'objet, au Québec, de questions relatives à la langue maternelle et à la langue "d'usage" des sujets de ces événements, et les données sont disponibles par division de recensement. Par ailleurs, nous connaissons, grâce au recensement de 1981, les lieux de résidence en 1976 et en 1981 des personnes présentes au Canada au moment du recensement de 1981 , et ce selon la langue maternelle et la "langue parlée à la maison" de ces dernières. Ceci permet donc d'analyser simultanément, pour une même période (la période censitaire 1976-1981) et pour un découpage territorial identique, le comportement de fécondité, de migration et de mortalité de chacun des principaux groupes linguistiques.

Bien entendu, idéalement, il eût été indiqué d'intégrer également la mobilité linguistique dans cette analyse multirégionale, c'est-à-dire de désagréger chaque région en "sous-régions linguistiques", de façon à pouvoir considérer en même temps la mobilité d'une région à l'autre et celle d'un groupe linguistique à $1^{\prime}$ autre. Aucune information $n^{\prime}$ est malheureusement disponible à cet égard pour la période 1976-1981. Notre analyse sera donc

* Institut national de la recherche scientifique (INRS-Urbanisation).

** Université du Québec à Chicoutimi.

Cet article est partiellement basé sur les résultats d'une recherche portant sur l'évolution récente de la situation démolinguistique au Québec, et financée par le Conseil de la langue française. Les auteurs tiennent à remercier Micheline Tremblay, qui a participé à l'élaboration des données, et François Tessier, qui a assuré le traitement informatique. 
limitée au seu1 comportement démographique des groupes 1inguistiques, et non au comportement linguistique de ces derniers. Et encore, en ce qui concerne le comportement migratoire, il nous faudra négliger la migration internationale : les données sur l'émigration internationale, par âge, région et groupe linguistique, sont soit inexistantes, soit non fiables, alors que l'immigration internationale, même si elle est un peu mieux connue, ne reflète évidemment guère le comportement de la population québécoise.

Dans une première étape, nous traiterons successivement des disparités linguistiques et régionales de la fécondité, de la mortalité et de la migration, chacune prise séparément. Dans une seconde étape, grâce à 1'approche multirégionale, nous analyserons ces trois phénomènes de façon simultanée. Au préalable, une brève présentation des données utilisées et du découpage spatio-temporel semble indiquée.

\section{LES DONNÉES}

Comme nous l'avons déjà mentionné, toutes les données utilisées concernent 1a période 1976-1981. Ce choix s'imposait puisque cette période est la seule pour laquelle nous disposons d'une désagrégation par région et par groupe linguistique pour chacun des trois phénomènes à analyser. Quant au découpage territorial, la nécessité d'avoir des chiffres statistiquement significatifs nous imposait un système régional qui ne soit pas trop désagrégé. L'importance de découper le Québec en régions linguistiquement significatives, de même que le souci de pouvoir comparer nos résultats à ceux obtenus par d'autres chercheurs pour des périodes antérieures'l, sont également à la base de notre choix.

Le découpage retenu comprend six grandes régions, soit l'Outaouais, la région étendue de Montréal, les Cantons de l'Est, l'Intérieur, la Gaspésie et le Nord (nous présentons en annexe les divisions de recensement comprises dans chacune de ces régions). En plus de correspondre à des régions bien identifiées du Québec, un tel découpage comporte l'avantage de regrouper au sein d'une même région la plupart des divisions de recensement où la proportion d'anglophones atteint ou dépasse dix pour cent. Chaque région compte par ailleurs en 1981 une population d'au moins 200000 habitants, est constituée de divisions entières de recensement, et n'est pas traversée par une frontière provinciale. Comme on le verra plus loin, même avec des critères aussi larges, il n'a pas toujours été possible d'obtenir, pour chaque groupe linguistique et pour chaque phénomène démographique, des chiffres significatifs. Afin de clore le système, nous avons évidemment ajouté une septième région, comprenant le reste du Canada.

1. Plus particulièrement, il nous a paru important d'assurer la comparabilité avec l'étude de Lachapelle et Henripin (1980). 
A nouveau par souci d'obtenir des résultats statistiquement significatifs, il nous a fallu limiter notre désagrégation par âge à 18 groupes d'âge (de $0-4$ ans à 85 ans et plus), et éliminer toute distinction de sexe (sauf pour la fécondité, où les données utilisées sont réparties suivant 1 'âge de $1 \mathrm{a}$ mère). Ceci limite évidemment quelque peu 1'analyse de la mortalité, mais n'affecte guère celle de la migration et de la fécondité.

Les données migratoires nécessaires à une analyse multirégionale sont des données sur les flux origine-destination par groupe d'âge. Seul le recensement, en 1 'occurrence celui de 1981 , nous fournit cette information. Celle-ci est disponible aussi bien selon la langue maternelle des personnes migrantes que selon 1a "langue parlée à la maison" ${ }^{2}$. Quant à la fécondité, les registres de l'état civil fournissent (depuis 1975) le nombre de naissances par région (division de recensement) et par âge de $1 \mathrm{a}$ mère, et ce aussi bien selon la langue maternelle de cette dernière que selon sa "langue d'usagel. Par contre, le nombre de décès, par région et âge, également obtenu à partir des registres de l'état civil, n'est désagrégé que selon la "langue d'usage" du décédé ${ }^{3}$. Ceci nous amène donc à définir nos groupes linguistiques selon la "langue d'usage", considérée ici comme synonyme de la "langue parlée à la maison". Trois groupes seront distingués : le groupe "français", le groupe "anglais", et le groupe "autre". La contrainte statistique n'est pas la seule justification du choix de la langue d'usage comme variable linguistique. On peut en effet raisonnablement supposer que la langue d'usage est plus significative pour l'étude du comportement démographique que ne l'est la langue maternelle.

2. Sauf dans le cas des personnes nées durant la période censitaire. Pour obtenir cette information, nous avons utilisé la question sur le lieu de naissance : les enfants de 0-4 ans résidant en 1981 dans une région autre que leur région de naissance, sont évidemment des migrants. Comme cependant les données ne sont disponibles que selon la province de naissance, nous avons dû estimer (à partir des flux migratoires du groupe 20-34 ans) la répartition selon la région d'origine des enfants de 0-4 ans ayant émigré du Québec vers le reste du Canada.

3. Les données de l'état civil relatives à la répartition linguistique des naissances et des décès ne sont pas exemptes de problèmes, surtout pour les premières années de la période. Dans le cadre de ce bref article, il ne nous est évidemment pas possible d'en discuter. Voir à ce sujet la très bonne présentation qu'en fait Tremblay (1983). Tout comme ce dernier, dans le cas des décès nous avons réparti les "inconnus" au prorata de la répartition connue. Une méthode plus complexe a été utilisée dans le cas des naissances : elle est basée sur les indices relatifs observés au recensement de 1981 chez les femmes des divers groupes linguistiques en matière de nombre d'enfants mis au monde. En outre, lorsque les données de l'état civil n'étaient disponibles que par année civile, nous avons ajusté les chiffres pour pouvoir passer aux années censitaires; cet ajustement a été effectué en utilisant les données mensuelles fournies par Statistique Canada. 
En adoptant la langue d'usage comme définition de nos groupes linguistiques, nous nous trouvons cependant devant un problème particulièrement délicat lorsqu'il s'agit du calcul de la population "soumise au risque". Si la population de 1981 est connue aussi bien selon la langue d'usage que selon la langue maternelle, seule cette dernière variable a été recueillie en 1976. Nous avons donc estimé, pour chaque région et pour chaque groupe d'âge, la population de chaque langue d'usage en 1976, en partant de la population recensée en 1981, en en retranchant les immigrants (et les naissances s'il y a lieu), et en y ajoutant les décès et les émigrants. Cette estimation ne devrait affecter que marginalement la valeur des taux, puisqu'une erreur dans 1'estimation de la population de 1976 se trouve "noyée" dans la moyenne des chiffres de 1976 et 1981. Les taux du groupe "autre" sont cependant plus sujets à caution que les taux des groupes français et anglais, à cause de l'importance de la migration internationale (moins bien estimée) pour ce groupe 1inguistique. En outre, il ne faudrait pas oublier le fait que le sous-dénombrement de la population peut varier sensiblement d'un âge à l'autre, d'une région à l'autre et d'un groupe linguistique à l'autre. En l'absence d'informations adéquates, nous n'avons cependant pas pu tenir compte de ce problème.

D'autres limites peuvent affecter de façon plus ou moins importante la valeur des résultats que nous présentons ici. Les problèmes de déclaration de langue qui caractérisent les recensements sont importants lorsqu'il s'agit de mesurer 1'intensité des transferts linguistiques, mais ils n'ont pas un impact aussi grand sur le type de mesures utilisées ici (Albert et Harrison, 1984). Le problème majeur réside probablement dans le fait d'utiliser simultanément, dans une même mesure, les données provenant de deux sources différentes, à savoir l'état civil et les recensements, dont les biais, même légers, ne sont peut-être pas comparables et pourraient même se multiplier. Nous n'avons malheureusement pas de moyen pour évaluer 1'importance de ce type de problème, sinon la cohérence même des résultats que nous obtenons.

Il n'est évidemment pas possible, dans le cadre de cet article, de présenter l'ensemble des chiffres qui ont servi de base à notre analyse. Pour permettre cependant au lecteur d'évaluer, fut-ce de façon approximative, la valeur du matériau statistique utilisé, nous présentons dans le tableau 1 les chiffres totaux (tous âges) des principales variables démographiques, désagrégées selon la région et le groupe linguistique.

\section{LA FÉCONDITE ET LA MORTALITE}

La fécondité des différents groupes linguistiques est depuis longtemps relativement bien connue. En effet, grâce aux recensements, on pouvait connaître le nombre moyen d'enfants mis au monde par les femmes non célibataires, suivant leur langue maternelle et, depuis 1971, selon leur langue d'usage. Les données avaient permis de constater la surfécondité historique du groupe français par rapport aux deux autres groupes, et - plus récemment - la surfécondité du groupe allophone. 
Tableau 1

Nombre total de naissances, décès et migrants, et population moyenne de la période 1976-1981, selon la langue d'usage et la région (en milliers)

\section{Population Naissances Décès Émigrants Immigrants} moyenne

\begin{tabular}{|c|c|c|c|c|c|}
\hline \multicolumn{6}{|c|}{ A- Groupe francais } \\
\hline Outaouais & 188 & 16 & 6 & 19 & 21 \\
\hline Montréal & 2247 & 167 & 81 & 115 & 132 \\
\hline Cantons & 280 & 25 & 9 & 29 & 29 \\
\hline Intérieur & 1544 & 123 & 55 & 107 & 110 \\
\hline Gaspésie & 325 & 27 & 11 & 34 & 26 \\
\hline Nord & 561 & 56 & 15 & 58 & 42 \\
\hline Canada-Québec & 647 & 55 & 24 & 42 & 44 \\
\hline Québec & 5146 & 414 & 178 & 44 & 42 \\
\hline \multicolumn{6}{|c|}{ B- Groupe anglais } \\
\hline Outaouais & 49 & 3 & 1 & 13 & 9 \\
\hline Montréal & 691 & 37 & 24 & 137 & 33 \\
\hline Cantons & 47 & 3 & 3 & 8 & 5 \\
\hline Intérieur & 37 & 2 & 1 & 14 & 5 \\
\hline Gaspésie & 16 & 1 & 1 & 4 & 2 \\
\hline Nord & 25 & 2 & 0 & 11 & 3 \\
\hline Canada-Québec & 15232 & 1177 & 531 & 38 & 169 \\
\hline Québec & 864 & 48 & 30 & 169 & 38 \\
\hline \multicolumn{6}{|l|}{ C- Groupe autre } \\
\hline Outaouais & 4 & 0 & 0 & 1 & 0 \\
\hline Montréal & 265 & 20 & 7 & 10 & 3 \\
\hline Cantons & 3 & 0 & 0 & 0 & 0 \\
\hline Intérieur & 8 & 1 & 0 & 1 & 1 \\
\hline Gaspésie & 1 & 0 & 0 & 0 & 0 \\
\hline Nord & 19 & 3 & 1 & 1 & 1 \\
\hline Canada-Québec & 1416 & 106 & 73 & 2 & 11 \\
\hline Québec & 300 & 24 & 8 & 11 & 2 \\
\hline
\end{tabular}

Sources : Statistique Canada, compilations spéciales du recensement de 1981 , pour les données relatives aux migrations; Ministère des affaires sociales, Registre de la population, Québec, pour les données relatives aux naissances et décès. 
Les données transversales de 1976-1981, présentées dans le tableau 2, confirment cette surfécondité du groupe "autre», tout en permettant d'évaluer l'ampleur de la sous-fécondité du groupe anglais. Pour 1'ensemble du Québec, seul le groupe "autre" atteint un taux brut de reproduction (sexes combinés) qui permet (tout juste) d'assurer le renouvellement de la population, alors qu'avec un taux de 0,67 le groupe anglais connait un des taux les plus bas que $l^{\prime}$ 'on puisse observer de par le monde. Le taux du groupe français se situe à mi-chemin entre ceux des deux autres groupes.

C'est dans toutes les régions du Québec que le groupe anglais manifeste la fécondité la plus basse (toujours en deçà du seuil de renouvellement), et le groupe "autre" la fécondité la plus élevée (toujours au-dessus du seuil de renouvellement, sauf à Montréal). Les disparités régionales sont importantes pour les groupes "autre" (de 0,98 à 1,83) et anglais (de 0,63 à $1,01)$, mais relativement faibles pour le groupe français $(0,80$ à 1,05$)$. Le fait que les écarts régionaux sont beaucoup plus prononcés pour le groupe "autre" est sans nul doute lié aux différences régionales dans la composition de ce groupe. A Montréal, ce groupe comprend essentiellement des immigrants, tandis que dans la région Nord, ainsi qu'en Gaspésie, il est composé en bonne partie de personnes d'origine indienne ou inuit. I1 importe en outre de souligner que la sous-fécondité du groupe anglais et la surfécondité du groupe "autre" s'observent non seulement pour toutes les régions mais encore pour tous les groupes d'âge (sauf trois cas négligeables parce que portant sur des effectifs statistiquement non significatifs) ${ }^{4}$.

Les différences linguistiques et régionales dans l'âge moyen des mères ne sont guère importantes. Elles disparaissent une fois que l'on élimine les différences dans la structure par âge de la population. A l'intérieur d'une même région, les âges moyens standardisés ne varient guère d'un groupe linguistique à l'autre, et ils sont identiques pour l'ensemble du Québec; les disparités régionales sont également très faibles ( 1 'écart maximum est d'environ un an).

Jusqu'à récemment, la mesure du caractère différentiel de la mortalité des divers groupes linguistiques était limitée, vu l'absence de données, à des estimations indirectes basées soit sur la variable géographique, soit sur la variable ethnique. Dans $1^{\prime}$ un et $1^{\prime}$ autre cas, les travaux faisaient ressortir la surmortalité du groupe français. Cette conclusion se trouve confirmée par l'étude de Tremblay (1983) qui, utilisant les données de l'état civil désagrégées suivant la langue d'usage du décédé, a calculé ce qui constitue à notre connaissance les premières tables de mortalité pour

4. A titre indicatif, nous avons également présenté dans le tableau 2 les taux relatifs au reste du Canada. Ces chiffres étant basés sur des estimations (il n'y a pas d'enregistrement des naissances selon la langue d'usage de la mère), nous ne les commenterons pas. 
Tableau 2

Les disparités lingulstıques et régionales de la fécondité, 1976-1981

\begin{tabular}{|c|c|c|c|c|c|c|c|c|c|c|c|}
\hline \multirow{2}{*}{\multicolumn{2}{|c|}{$\begin{array}{l}\text { égion et } \\
\text { roupe } \\
\text { ingulstique }\end{array}$}} & \multicolumn{6}{|c|}{ Taux de fécondité par âge ${ }^{b}(\chi)$} & \multirow{2}{*}{$\begin{array}{l}\text { Taux brut } \\
\text { de } \\
\text { reproduction c }\end{array}$} & \multirow{2}{*}{$\begin{array}{l}\text { Taux brut } \\
\text { de } \\
\text { natalite } \\
(\text { en } 0 / 00)\end{array}$} & \multicolumn{2}{|c|}{ Age moyen de la mère } \\
\hline & & $15-19$ & $20-24$ & $25-29$ & $30-34$ & $35-39$ & $40-44$ & & & Observé & Standardisé \\
\hline utaoua is & $\begin{array}{l}-\mathbf{F} \\
-\mathbf{A} \\
-\mathbf{0}\end{array}$ & $\begin{array}{l}1,3 \\
1,2 \\
2,0\end{array}$ & $\begin{array}{l}5,7 \\
5,2 \\
6,8\end{array}$ & $\begin{array}{r}6,5 \\
5,4 \\
12,0\end{array}$ & $\begin{array}{l}2,9 \\
2,7 \\
3,4\end{array}$ & $\begin{array}{l}0,7 \\
0,6 \\
1,1\end{array}$ & $\begin{array}{l}0,2 \\
0,2 \\
0,3\end{array}$ & $\begin{array}{l}0,87 \\
0,77 \\
1,28\end{array}$ & $\begin{array}{l}16,7 \\
12,6 \\
22,9\end{array}$ & $\begin{array}{l}26,0 \\
26,3 \\
26,5\end{array}$ & $\begin{array}{l}26,5 \\
26,5 \\
26,6\end{array}$ \\
\hline ontréal & $\begin{array}{l}-\mathbf{F} \\
-\mathbf{A} \\
-0\end{array}$ & $\begin{array}{l}0,8 \\
0,6 \\
1,0\end{array}$ & $\begin{array}{l}4,3 \\
3,4 \\
5,2\end{array}$ & $\begin{array}{l}6,4 \\
4,9 \\
7,6\end{array}$ & $\begin{array}{l}3,4 \\
2,8 \\
4,5\end{array}$ & $\begin{array}{l}1,0 \\
0,8 \\
1,1\end{array}$ & $\begin{array}{l}0,2 \\
0,1 \\
0,2\end{array}$ & $\begin{array}{l}0,80 \\
0,63 \\
0,98\end{array}$ & $\begin{array}{l}14,9 \\
10,6 \\
14,8\end{array}$ & $\begin{array}{l}27,0 \\
27,0 \\
27,6\end{array}$ & $\begin{array}{l}27,5 \\
27,5 \\
27,5\end{array}$ \\
\hline antons & $\begin{array}{l}-F \\
-A \\
-0\end{array}$ & $\begin{array}{l}1,1 \\
1,0 \\
1,7\end{array}$ & $\begin{array}{l}5,9 \\
4,7 \\
6,9\end{array}$ & $\begin{array}{r}7,4 \\
6,6 \\
10,9\end{array}$ & $\begin{array}{l}3,6 \\
3,1 \\
5,1\end{array}$ & $\begin{array}{l}0,9 \\
0,8 \\
0,7\end{array}$ & $\begin{array}{l}0,1 \\
0,1 \\
0,2\end{array}$ & $\begin{array}{l}0.95 \\
0,82 \\
1,28\end{array}$ & $\begin{array}{l}17,5 \\
11,4 \\
18,9\end{array}$ & $\begin{array}{l}26,3 \\
26,5 \\
26,6\end{array}$ & $\begin{array}{l}26,9 \\
27,0 \\
26,9\end{array}$ \\
\hline ntérieur & $\begin{array}{l}-F \\
-A \\
-0\end{array}$ & $\begin{array}{l}0,7 \\
0,6 \\
1,0\end{array}$ & $\begin{array}{l}4,6 \\
3,9 \\
6,7\end{array}$ & $\begin{array}{l}7,4 \\
5,8 \\
9,9\end{array}$ & $\begin{array}{l}3,9 \\
3,7 \\
5,4\end{array}$ & $\begin{array}{l}1,1 \\
1,0 \\
1,1\end{array}$ & $\begin{array}{l}0,2 \\
0,1 \\
0,3\end{array}$ & $\begin{array}{l}0,89 \\
0,75 \\
1,22\end{array}$ & $\begin{array}{l}16,0 \\
12,5 \\
21,2\end{array}$ & $\begin{array}{l}27,1 \\
27,4 \\
27,0\end{array}$ & $\begin{array}{l}27,7 \\
27,9 \\
27,5\end{array}$ \\
\hline aspésie & $\begin{array}{l}-F \\
-A \\
-0\end{array}$ & $\begin{array}{l}0,9 \\
1,0 \\
0,5\end{array}$ & $\begin{array}{l}5,2 \\
5,5 \\
8,6\end{array}$ & $\begin{array}{r}7,5 \\
6,6 \\
14,2\end{array}$ & $\begin{array}{l}4,0 \\
4,2 \\
3,7\end{array}$ & $\begin{array}{l}1,2 \\
1,3 \\
2,3\end{array}$ & $\begin{array}{l}0,3 \\
0,3 \\
0,4\end{array}$ & $\begin{array}{l}0,95 \\
0,94 \\
1,48\end{array}$ & $\begin{array}{l}16,4 \\
13,8 \\
27,1\end{array}$ & $\begin{array}{l}26,8 \\
26,8 \\
26,5\end{array}$ & $\begin{array}{l}27,6 \\
27,5 \\
27,5\end{array}$ \\
\hline ord & $\begin{array}{l}-\mathbf{F} \\
-\mathbf{A} \\
-0\end{array}$ & $\begin{array}{l}1,4 \\
1,3 \\
2,5\end{array}$ & $\begin{array}{r}6,6 \\
6,2 \\
11,9\end{array}$ & $\begin{array}{r}7,7 \\
7,8 \\
13,4\end{array}$ & $\begin{array}{l}4,0 \\
3,7 \\
6,3\end{array}$ & $\begin{array}{l}1,1 \\
0,9 \\
2,0\end{array}$ & $\begin{array}{l}0,2 \\
0,2 \\
0,4\end{array}$ & $\begin{array}{l}1,05 \\
1,01 \\
1,83\end{array}$ & $\begin{array}{l}20,1 \\
17,2 \\
26,3\end{array}$ & $\begin{array}{l}26,2 \\
26,4 \\
25,7\end{array}$ & $\begin{array}{l}27,0 \\
26,9 \\
26,8\end{array}$ \\
\hline uébec & $\begin{array}{l}-F \\
-A \\
-0\end{array}$ & $\begin{array}{l}0,9 \\
0,7 \\
1,2\end{array}$ & $\begin{array}{l}4,9 \\
3,6 \\
5,8\end{array}$ & $\begin{array}{l}7,0 \\
5,1 \\
8,2\end{array}$ & $\begin{array}{l}3,6 \\
2,9 \\
4,6\end{array}$ & $\begin{array}{l}1,0 \\
0,8 \\
1,1\end{array}$ & $\begin{array}{l}0,2 \\
0,1 \\
0,2\end{array}$ & $\begin{array}{l}0,88 \\
0,67 \\
1,05\end{array}$ & $\begin{array}{l}16,1 \\
11,1 \\
15,9\end{array}$ & $\begin{array}{l}26,9 \\
26,9 \\
27,4\end{array}$ & $\begin{array}{l}27,4 \\
27,4 \\
27,4\end{array}$ \\
\hline $\begin{array}{l}\text { anada } \\
\text { olns } \\
\text { uébec }\end{array}$ & $\begin{array}{l}-\mathbf{F} \\
-\mathbf{A} \\
-0\end{array}$ & $\begin{array}{l}2,0 \\
1,7 \\
2,0\end{array}$ & $\begin{array}{l}5,9 \\
5,3 \\
6,0\end{array}$ & $\begin{array}{l}6,8 \\
6,1 \\
7,0\end{array}$ & $\begin{array}{l}3,7 \\
3,2 \\
4,0\end{array}$ & $\begin{array}{l}1,1 \\
0,9 \\
1,1\end{array}$ & $\begin{array}{l}0,2 \\
0,2 \\
0,2\end{array}$ & $\begin{array}{l}0,98 \\
0,87 \\
1,02\end{array}$ & $\begin{array}{l}17,0 \\
15,5 \\
15,0\end{array}$ & $\begin{array}{l}25,9 \\
26,0 \\
27,0\end{array}$ & $\begin{array}{l}26,6 \\
26,6 \\
26,7\end{array}$ \\
\hline anada & $\begin{array}{l}\text { - F } \\
\text { - A } \\
\text { - O } \\
\text { - Total }\end{array}$ & $\begin{array}{l}1,0 \\
1,7 \\
1,8 \\
1,5\end{array}$ & $\begin{array}{l}5,0 \\
5,2 \\
6,0 \\
5,2\end{array}$ & $\begin{array}{l}7,0 \\
6,0 \\
7,2 \\
6,3\end{array}$ & $\begin{array}{l}3,6 \\
3,2 \\
4,1 \\
3,4\end{array}$ & $\begin{array}{l}1,0 \\
0,9 \\
1,1 \\
1,0\end{array}$ & $\begin{array}{l}0,2 \\
0,2 \\
0,2 \\
0,2\end{array}$ & $\begin{array}{l}0,89 \\
0,86 \\
1,02 \\
0,88\end{array}$ & $\begin{array}{l}16,2 \\
15,2 \\
15,2 \\
15,5\end{array}$ & $\begin{array}{l}26,7 \\
26,0 \\
27,0 \\
26,3\end{array}$ & $\begin{array}{l}27,3 \\
26,7 \\
26,9 \\
26,8\end{array}$ \\
\hline
\end{tabular}

Les groupes "français», kanglais» et (autrè sont désignés respectivement par les sigles F, A et 0 .

Le taux de fécondité de chaque groupe d'âge est obtenu en divisant un cinquième du nombre de naissances observées dans ce groupe d'âge (de la mère) entre 1976 et 1981 par la moyenne arithmétique des effectifs de ce groupe d'âge en 1976 et 1981 .

Le taux brut de reproduction (sexes combinés) est obtenu en sommant les taux de fécondité par âge et en multipliant le résultat par cinq (le nombre d'années de chaque groupe d'âge).

Le taux brut de natalité est obtenu en divisant le cinquième du nombre total de nalssances observées entre 1976 et 1981, par la moyenne arithmétique des effectifs de population en 1976 et 1981.

S1 1 'on désigne par $F$ le taux de fécondité du groupe d'âge $x$ à $x+4$, et par p le pourcentage de naissances du groupe d'âge $x$ à $x+4^{x}$ dans le total des nalssances, alors 1'âge moyen Xóbservé de la mère est égal à $\frac{1}{100} \sum_{x}(x+2,5) P_{x}$, et l'âge moyen ustandardisé" (pour éliminer les différences dans la structure par âge de la population) est égal à $\sum_{\mathbf{x}}\left[\frac{F_{X}}{\sum_{\mathbf{X}} F_{X}}(x+2,5)\right]$. 
les groupes linguistiques au Québec. Ces tables, établies pour la période 1980-1982 (donc, a priori, avec des données plus fiables que celles utilisées ici), font également ressortir la sous-mortalité du groupe "autre".

Les résultats que nous avons obtenus pour la période 1976-1981, présentés dans le tableau 3, rejoignent ceux obtenus par Tremblay : l'espérance de vie à la naissance d'un Québécois du groupe français est de quatre ans inférieure à celle d'un Québécois du groupe anglais, et de près de six ans inférieure à celle d'un Québécois du groupe "autre". Il s'agit ici. d'une espérance de vie calculée sans distinction de sexe : des chiffres non présentés ici révèlent que pour les nouveau-nés de sexe masculin, l'écart entre groupe français et groupe "autre" est de plus de sept ans (il est de moins de cinq ans pour les nouveau-nées).

La surmortalité de la population du groupe français par rapport au groupe "autre" apparaît à tout âge. A titre illustratif, nous présentons dans le tableau 3 les taux observés pour les groupes d'âge de $0-4$ ans, 20-24 ans, 60-64 ans et 75-79 ans. Le taux de mortalité relativement élevé pour le groupe "autre" d'âge $0-4$ ans est la seule exception que 1 'on puisse observer tout au long de la courbe de mortalité par âge : pour le groupe "autre" comme pour le groupe français, la mortalité infantile reste relativement élevée (elle est en fait près du double de celle du groupe anglais). C'est après 20 ans, lorsque les taux de mortalité s'élèvent, que la sous-mortalité du groupe "autrel et la surmortalité du groupe français apparaissent. L'écart entre le taux de ces deux groupes est du simple au double à 20-24 ans comme à 60-64 ans; à 75-79 ans, la probabilité de décès d'un francophone du Québec est encore d'environ 50 pour cent plus élevée que celle d'un allophone (près de 60 pour cent à Montréal).

Une comparaison interrégionale des taux de mortalité par âge et groupe linguistique n'est guère significative, à cause des nombreux chiffres peu élevés. Seule une comparaison entre la région de Montréal et le reste du Quëbec semble pouvoir se justifier. Il en ressort qu'aux âges jeunes les Montréalais ont un risque de décès moindre que les autres citoyens du Québec, et ce quel que soit le groupe linguistique, mais qu'aux âges avancés, les chances de survie sont plus favorables en dehors de Montréal (les Montréalais du groupe "autre" ont cependant toujours un avantage sur ceux du reste du Québec, même aux âges avancés).

Le net avantage qui caractérise le groupe "autre" par rapport aux deux autres groupes doit certes être vu comme un effet sélectif de l'immigration internationale (les candidats à l'immigration internationale sont soumis à des examens médicaux souvent rigoureux). On peut cependant se demander si cet effet ne se trouve pas amplifié par le problème de déclaration de $1 a$ langue des décédés (il revient au médecin qui constate le décès d'inscrire la langue d'usage du décédé, ce qui pourrait conduire à sous-estimer le nombre de décédés de langue autre, au profit de ceux de langue française et surtout anglaise). 


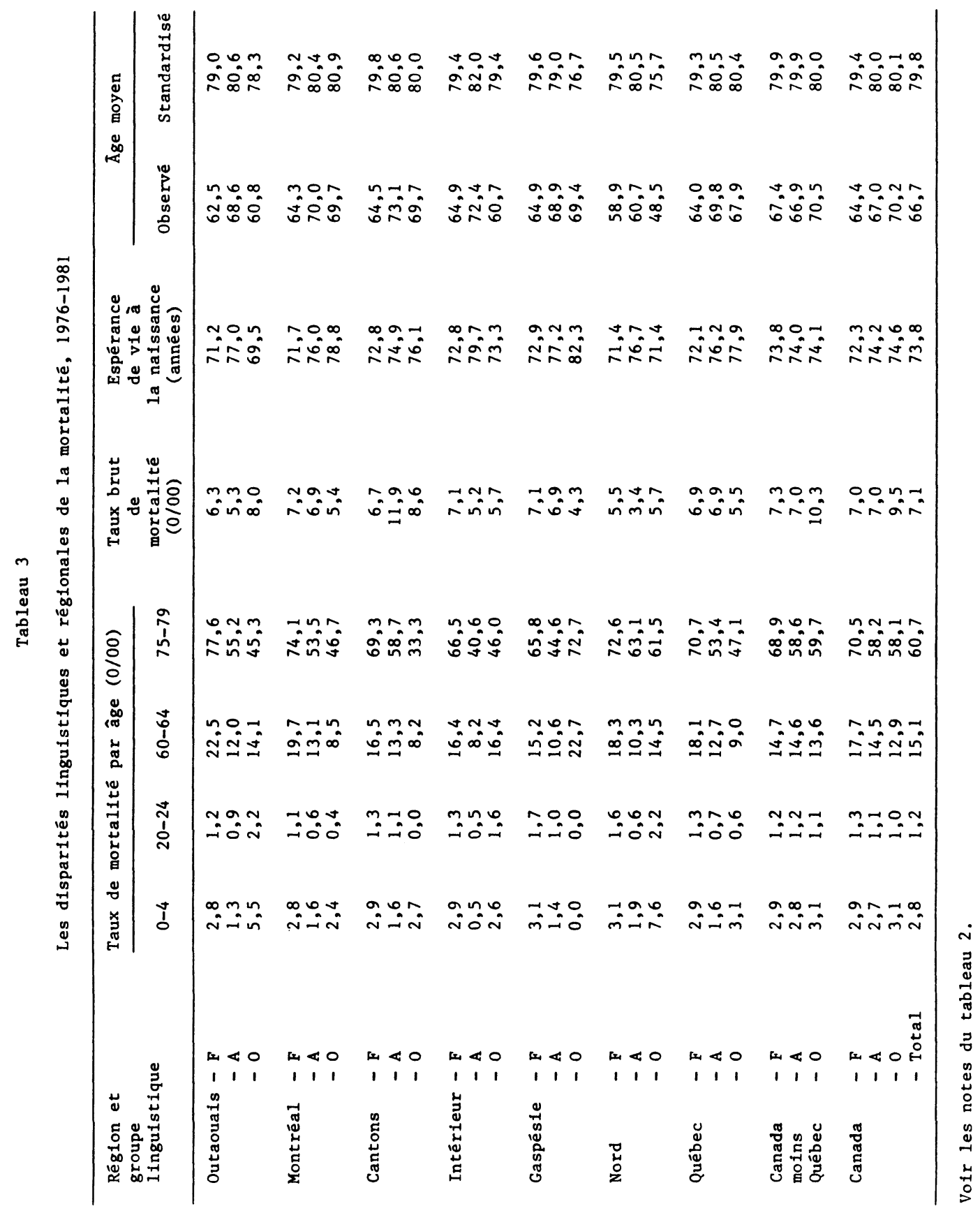


Dans toutes les régions (à l'exception de la Gaspésie où les effectifs sont cependant trop faibles pour donner des résultats significatifs), 1'âge moyen observé au décès est le plus élevé pour le groupe anglais. D'une façon générale, c'est dans les Cantons de l'Est que l'âge moyen au décès est le plus élevé (sauf pour le groupe français), alors que, quel que soit le groupe linguistique, c'est dans la région Nord que cet âge est le plus bas. Toutefois, 1 'essentiel des disparités régionales et linguistiques s'explique ici par les différences dans la structure par âge. En effet, pour 1'ensemble du Québec comme pour la région de Montréal (seule région où les effectifs sont suffisamment élevés pour chacun des groupes linguistiques pour conduire à des résultats significatifs), les différences dans l'âge moyen standardisé sont minimes.

Les différences dans la structure par âge des divers groupes sont également à l'origine de taux bruts de natalité et de mortalité qui ne reflètent pas la même image des différences dans les comportements démographiques selon la langue. Ainsi, la structure par âge du groupe anglais est nettement plus vieille que celle des deux autres groupes, en particulier dans les Cantons de l'Est, ce qui a pour effet d'augmenter sensiblement les taux bruts de mortalité de ce groupe.

La différence entre les taux bruts de natalité (tableau 2) et les taux bruts de mortalité (tableau 3) permet de connaître les taux annuels moyens d'accroissement naturel. Quelle que soit la région, le taux d'accroissement naturel du groupe anglais est toujours le plus bas et celui du groupe "autre" toujours le plus élevé (sauf dans les Cantons de 1'Est où cependant l'effectif du groupe "autre" est trop faible pour donner des taux significatifs). On pourra constater que pour 1'ensemble du Québec, le groupe anglais connaît un taux d'accroissement naturel inférieur à la moitié de celui des groupes français et "lautre". On remarquera aussi que le groupe anglais a connu (entre 1976 et 1981) une croissance naturelle négative dans les Cantons de l'Est, et une croissance naturelle quasi nulle à Montréal $(0,37$ pour cent par an).

\section{LA MIGRATION}

Trop souvent encore, l'analyse de la migration se limite à l'examen de la migration nette ou, au mieux, du flux total d'émigration et d'immigration. C'est comme si l'analyse de la fécondité et de la mortalité était limitée à 1 'étude de $1^{\prime}$ accroissement naturel ou du nombre total de naissances et de décès. Dans le cadre d'une analyse multirégionale, où l'on considère simultanément les interrelations de tous les phénomènes démographiques entre toutes les régions, il est impératif de prendre en compte les flux origine-destination, désagrégés par âge et, en ce qui nous concerne, par groupe linguistique. Les tableaux 4-A, 4-B et 4-C présentent les caractéristiques principales de ces flux interrégionaux. Par souci de brièveté, nous ne pourrons cependant présenter ici les taux spécifiques par âge; seul l'âge moyen des migrants fournira une indication de la structure par âge des flux de migration. Comme on pourra le vérifier 
Tableau 4-A

Taux de migration interrégionale $(e n-0 / 00)^{a}$ et âge moyen des migrants, 1976-1981. Groupe français

\begin{tabular}{|c|c|c|c|c|c|c|c|c|c|c|c|c|c|c|c|c|c|}
\hline \multirow[b]{3}{*}{ De : } & \multirow[t]{3}{*}{ Vers : } & \multirow[t]{3}{*}{0} & \multirow[t]{3}{*}{$\mathbf{M}$} & \multirow[t]{3}{*}{ C } & \multirow[t]{3}{*}{ I } & \multirow[t]{3}{*}{ G } & \multirow[t]{3}{*}{$\mathbf{N}$} & \multirow[t]{3}{*}{$C-Q$} & \multicolumn{3}{|c|}{ Taux totaux $($ en $0 / 00)$} & \multicolumn{2}{|c|}{ Tx migrapr. ${ }^{c}$} & \multicolumn{4}{|c|}{ Äge moyen ${ }^{d}$} \\
\hline & & & & & & & & & \multirow{2}{*}{$\mathrm{EM}^{\mathrm{b}}$} & \multirow{2}{*}{ IM } & \multirow{2}{*}{ NET } & \multirow{2}{*}{ EM } & \multirow{2}{*}{ IM } & \multicolumn{4}{|c|}{ EM IM } \\
\hline & & & & & & & & & & & & & & obs. & Stand. & obs. & Stand. \\
\hline Outaouais ( 0 ) & & - & 6,8 & 0,5 & 3,4 & 0,6 & 1,7 & 7,0 & 20,1 & 22,0 & 1,9 & 1,40 & 1,59 & 23,6 & 32,1 & 20,6 & 30,4 \\
\hline Montréal (M) & & 0,4 & - & 1,1 & 5,3 & 0,8 & 1,2 & 1,5 & 10,3 & 11,8 & 1,5 & 0,78 & 0,85 & 25,8 & 33,4 & 23,8 & 31,2 \\
\hline Cantons (C) & & 0,4 & 9,9 & - & 7,1 & 0,5 & 1,3 & 1,5 & 20,6 & 20,6 & $-0,0$ & 1,43 & 1,50 & 22,9 & 30,6 & 24,2 & 33,2 \\
\hline Intérieur (I) & & 0,3 & 8,1 & 1,4 & - & 1,0 & 1,8 & 1,1 & 13,8 & 14,3 & 0,5 & 0,98 & 1,04 & 23,4 & 30,6 & 24,6 & 31,8 \\
\hline Gaspésie（G） & & 0,2 & 7,5 & 0,7 & 7,2 & - & 3,8 & 1,7 & 21,1 & 16,0 & $-5,1$ & 1,45 & 1,13 & 21,9 & 29,0 & 23,0 & 28,8 \\
\hline Nord (N) & & 0,7 & 7,7 & 0,9 & 6,9 & 2,0 & - & 2,5 & 20,7 & 15,0 & $-5,7$ & 1,44 & 0,97 & 23,2 & 32,9 & 20,9 & 27,9 \\
\hline Canada-Québec $(C-Q)$ & & 3,3 & 4,9 & 0,4 & 2,1 & 0,7 & 1,7 & - & 13,1 & 13,7 & 0,6 & 0,91 & 0,97 & 21,9 & 26,8 & 25,8 & 31,8 \\
\hline
\end{tabular}

Tableau 4-B

Taux de migration interrégionale $(\text { en } 0 / 00)^{a}$ et âge moyen des migrants, 1976-1981. Groupe anglais

\begin{tabular}{|c|c|c|c|c|c|c|c|c|c|c|c|c|c|c|c|c|c|}
\hline \multirow{3}{*}{ De : } & \multirow[t]{3}{*}{ Vers : } & \multirow[t]{3}{*}{0} & \multirow[t]{3}{*}{ M } & \multirow[t]{3}{*}{$\mathrm{C}$} & \multirow[t]{3}{*}{ I } & \multirow[t]{3}{*}{ G } & \multirow[t]{3}{*}{$\mathrm{N}$} & \multirow[t]{3}{*}{$C-Q$} & \multicolumn{2}{|c|}{ Taux totaux } & \multicolumn{3}{|c|}{ 0/00) Tx migrapr. ${ }^{c}$} & \multicolumn{4}{|c|}{${ }_{\text {EM }}^{\text {Agge moyen }}{ }^{d}$} \\
\hline & & & & & & & & & \multirow{2}{*}{$\mathrm{EM}^{\mathrm{b}}$} & \multirow{2}{*}{ IM } & \multirow{2}{*}{ NET } & \multirow{2}{*}{ EM } & \multirow{2}{*}{ IM } & & \multicolumn{2}{|c|}{ IM } \\
\hline & & & & & & & & & & & & & & Obs. & Stand. & Obs. & Stand. \\
\hline Outaouais $(0)$ & & - & 2,4 & 0,2 & 0,2 & 0,1 & 0,2 & 49,5 & 52,7 & 36,6 & $-16,0$ & 3,82 & 2,60 & 26,0 & 32,7 & 18,0 & 23,0 \\
\hline Montréal (M) & & 0,1 & - & 0,7 & 0,5 & 0,1 & 0,2 & 38,1 & 39,7 & 9,5 & $-30,2$ & 3,23 & 0,72 & 30,6 & 38,3 & 25,9 & 30,7 \\
\hline Cantons (C) & & 0,3 & 9,0 & - & 1,0 & 0,1 & 0,2 & 25,4 & 35,9 & 19,3 & $-16,6$ & 2,91 & 1,64 & 28,3 & 32,2 & 31,3 & 35,5 \\
\hline Intérieur (I) & & 0,4 & 21,5 & 2,8 & - & 1,1 & 1,6 & 47,7 & 75,0 & 25,3 & $-49,8$ & 5,78 & 1,88 & 27,6 & 34,0 & 27,6 & 31,5 \\
\hline Gaspēsie (G) & & 0,1 & 11,0 & 0,2 & 3,4 & - & 1,1 & 35,9 & 51,8 & 19,3 & $-32,5$ & 4,04 & 1,46 & 26,7 & 34,9 & 23,1 & 28,0 \\
\hline Nord (N) & & 1,6 & 14,5 & 1,0 & 4,7 & 0,7 & - & 65,6 & 88,1 & 27,6 & $-60,4$ & 7,21 & 1,74 & 27,0 & 40,9 & 20,5 & 23,7 \\
\hline Canada-Québec $(C-Q)$ & & 0,1 & 0,3 & 0,0 & 0,0 & 0,0 & 0,0 & - & 0,5 & 2,2 & 1,7 & 0,04 & 0,18 & 22,9 & 28,3 & 29,7 & 39,7 \\
\hline
\end{tabular}


Tableau 4-C

Taux de migration interrégionale $(\text { en } 0 / 00)^{a}$ et âge moyen des migrants, 1976-1981. Groupe "autre"

\begin{tabular}{|c|c|c|c|c|c|c|c|c|c|c|c|c|c|c|c|c|c|}
\hline \multirow[b]{2}{*}{ De : } & \multirow[t]{2}{*}{ Vers : } & \multirow[t]{2}{*}{0} & \multirow[t]{2}{*}{ M } & \multirow[t]{2}{*}{$\mathrm{C}$} & \multirow[t]{2}{*}{ I } & \multirow[t]{2}{*}{ G } & \multirow[t]{2}{*}{$\mathrm{N}$} & \multirow[t]{2}{*}{$C-Q$} & \multicolumn{3}{|c|}{ Taux totaux (en $0 / 00)$} & \multicolumn{2}{|c|}{ Tx migrapr. ${ }^{c}$} & \multicolumn{4}{|c|}{ Age moyen ${ }^{d}$} \\
\hline & & & & & & & & & $E M^{b}$ & IM & NET & EM & IM & Obs. & Stand. & Obs. & Stand. \\
\hline Outaouais $(0)$ & & - & 3,3 & 0,8 & 0,3 & 0,0 & 1,0 & 19,8 & 25,1 & 16,0 & $-9,0$ & 1,85 & 1,11 & 28,9 & 35,3 & 22,0 & 28,2 \\
\hline Montréal (M) & & 0,0 & - & 0,1 & 0,3 & 0,0 & 0,1 & 7,0 & 7,6 & 2,4 & $-5,2$ & 0,68 & 0,20 & 38,2 & 46,7 & 30,2 & 38,2 \\
\hline Cantons (C) & & 0,0 & 23,4 & - & 1,9 & 0,0 & 0,8 & 8,7 & 34,8 & 18,9 & $-15,9$ & 2,68 & 1,71 & 28,6 & 31,4 & 42,0 & 49,8 \\
\hline Intérieur (I) & & 0,0 & 19,9 & 0,4 & - & 0,1 & 1,1 & 12,0 & 33,5 & 13,7 & $-19,8$ & 3,21 & 1,44 & 32,5 & 47,5 & 35,0 & 50,7 \\
\hline Gaspésie (G) & & 1,0 & 10,2 & 0,0 & 0,0 & - & 2,0 & 9,2 & 22,4 & 5,1 & $-17,3$ & 2,29 & 0,44 & 37,1 & 52,9 & 41,5 & 47,4 \\
\hline Nord $(\mathrm{N})$ & & 0,2 & 3,1 & 0,0 & 0,5 & 0,0 & - & 3,3 & 7,1 & 7,6 & 0,5 & 1,13 & 0,53 & 36,7 & 59,8 & 20,5 & 32,8 \\
\hline Canada-Québec $(C-Q)$ & & 0,0 & 0,2 & 0,0 & 0,0 & 0,0 & 0,1 & - & 0,4 & 1,5 & 1,2 & 0,03 & 0,13 & 26,8 & 30,6 & 38,0 & 43,0 \\
\hline
\end{tabular}

a. Les taux de migration de la région i à la région $j$ sont obtenus en divisant un cinquième des migrants de la région $i$ vers la région $\mathrm{j}$ recensés au recensement de 1981 pour la période 1976-1981, par la moyenne arithmétique de la population de 1976 et 1981 .

b. A cause des arrondissements, la somme des taux sur toutes les destinations n'est pas nécessairement égale au taux total d'émigration. Ces taux totaux d'émigration et d'immigration sont conceptuellement semblables aux taux bruts de natalité et de mortalité.

c. Le taux brut de migraproduction, obtenu par analogie avec le taux brut de reproduction, est calculé en sommant les taux de migration par âge et en multipliant le résultat par cinq (le nombre d'années de chaque groupe d'âge).

d. Volr la note e du tableau 2. Comme 1'âge du migrant est celui du migrant au moment du recensement, pour obtenir 1 'âge moyen du migrant au moment de la migration, 11 faut, en supposant l'uniformité temporelle de la migration au cours de la péríode 1976-1981, soustraíre 2,5 années aux chiffres d'âge moyen présentés dans ce tableau. 
ultérieurement, la prise en compte des différences régionales dans la structure par âge ne modifie cependant pas l'essentiel des résultats discutés ici.

I1 est facile de constater - et ce n'est guère une surprise - que le groupe anglais est celui qui, de loin, a manifesté la propension la plus forte à quitter sa région, et ce quelle que soit la région considérée : les taux annuels totaux d'émigration du groupe anglais varient de 3,6 pour cent (Cantons de 1'Est) à 8,8 pour cent (Nord), ceux du groupe "autre" de 0,7 pour cent (Nord) à 3,5 pour cent (Cantons de 1 'Est), et ceux du groupe français de 1,0 pour cent (Montréa1) à 2,1 pour cent (Gaspésie).

La très forte propension du groupe anglais à l'émigration est surtout due à l'émigration vers le reste du Canada : selon les régions, ce dernier a attiré annuellement de 2,5 pour cent (Cantons) à 6,6 pour cent (Nord) de 1a population du groupe anglais. En éliminant les chiffres (peu significatifs) de la région Nord, on peut constater que le reste du Canada a attiré entre 1976 et 1981 près d'un quart des anglophones de l'Outaouais et de l'Intérieur, près d'un cinquième de ceux de Montréal et de la Gaspésie, et plus d'un huitième de ceux des Cantons de 1 'Est.

On remarquera que la quasi-totalité des émigrants anglophones de Montréal et de 1'Outaouais sont partis pour une région en dehors du Québec. Au total, entre 1976 et 1981 , près de 20 pour cent des anglophones du Québec ont quitté la province pour une autre province du Canada, soit à un taux annuel moyen de 39 pour mille.

Pendant la même période, le taux annuel moyen d'émigration (vers le reste du Canada) des Québécols du groupe français était de 1,7 pour mille. Les anglophones se sont donc montrés 23 fois plus prompts à quitter le Québec que les francophones, ce qui représente un écart près de deux fois plus élevé que celui observé au cours des périodes censitaires précédentes : en 1966-1971 et 1971-1976, le taux du groupe anglais était environ 13 fois plus élevé que celui du groupe français (Lachapelle et Henripin, 1980 : 200-201).

Si, comparativement aux anglophones, les francophones du Québec font preuve d'une remarquable inertie spatiale dans leur relation avec le reste du Canada (à l'exception, très compréhensible, des francophones de l'Outaouais), il n'en va pas de même lorsqu'on considère les migrations à l'intérieur du Québec. Pour la grande part des paires origine-destination concernées, le groupe français manifeste une propension à la migration supérieure à celle du groupe anglais (les exceptions majeures sont celles relatives aux flux de l'Intérieur, de la Gaspésie et du Nord vers Montréal : dans ces cas, les anglophones ont des taux supérieurs à ceux des francophones).

Le groupe "autrel a manifesté une propension à quitter le Québec nettement moins forte que le groupe anglais : alors que le taux annuel moyen de ce dernier était de 39 pour mille, celui du groupe "autre" n'était que de 
7 pour mille, ce qui représente cependant encore quatre fois plus que le taux du groupe français ( 1,7 pour mille). Quelle que soit la région d'origine, le groupe "autre" s'est révélé nettement plus prompt à quitter le Québec que le groupe français. Si l'on tient également compte de la migration à 1'intérieur du Québec, le groupe "autre" reste encore généralement plus mobile que le groupe français. Ce n'est que dans le cas des régions de Montréal et du Nord que le groupe "autrell se montre globalement moins porté à l'émigration.

Les chiffres de 1976-1981 confirment une observation déjà faite par Lachapelle et Henripin (1980: 200) pour les périodes censitaires précédentes, à savoir que dans les relations migratoires entre le Québec et le reste du Canada, c'est le groupe linguistiquement minoritaire qui a tendance à émigrer le plus, et ce dans des proportions quasiment identiques. Nous avons vu que le groupe anglophone du Québec avait connu un taux d'émigration vers le reste du Canada 23 fois plus élevé que le groupe francophone du Québec. Dans 1'autre sens, les francophones du reste du Canada ont manifesté une propension annuelle à l'émigration vers le Québec 26 fois plus élevée que celle des anglophones du reste du Canada (13,1 pour mille contre 0,5 pour mille). Remarquons qu'avec un taux de 0,4 pour mille, les allophones du reste du Canada paraissent encore moins portés que les anglophones à émigrer vers le Québec.

On pourrait penser qu'une région "répulsive" est aussi une région peu attractive. Il n'en est rien. En règle générale, plus le taux d'émigration d'une région est élevé, plus le taux d'immigration dans cette région est élevé, et ce quel que soit le groupe linguistique. Cela est dû bien sûr au découpage territorial (les mouvements migratoires sont normalement plus importants lorsqu'il s'agit de "petites" régions, et ce quelle que soit la direction du flux). Mais cela s'explique également par une relation fonctionnelle entre émigration et immigration, c'est-à-dire par la migration de retour (les émigrants d'une région sont de futurs candidats à l'immigration dans cette même région).

Si l'on ajoute à ces considérations le fait que, pour un flux donné, les effectifs de population utilisés comme dénominateur dans le calcul des taux sont bien sûr différents selon qu'il s'agit du taux d'émigration de la région d'origine ou du taux d'immigration de la région de destination, on ne peut plus être tellement surpris que le taux annuel d'immigration des anglophones du reste du Canada au Québec soit supérieur au taux des francophones ( 2,2 pour mille contre 1,6 pour mille), alors que le taux d'émigration des francophones du reste du Canada vers le Québec est, comme nous l'avons vu, 26 fois plus élevé que celui des anglophones. Sauf pour Montréal et les Cantons de l'Est, on pourra également observer des taux d'immigration nettement plus élevés pour les anglophones que pour les francophones, lorsque les migrations entre régions du Québec sont également prises en compte. On notera enfin que, quelle que soit la région considérée, le taux d'immigration des allophones est toujours le plus faible. 
Lorsque 1 'on met en balance 1'émigration et l'immigration, il apparaît que le groupe linguistique français se retrouve dans une situation de quasi-équilibre spatial. Dans ses relations migratoires avec le reste du Canada, le groupe français du Québec n'a perdu annuellement (entre 1976 et 1981) que 0,01 pour cent de sa population (en chiffres absolus, moins de 400 personnes par an). Même au niveau régional, c'est-à-dire en tenant compte des migrations entre les régions du Québec, on peut observer un équilibre remarquable des échanges migratoires : les taux annuels d'accroissement migratoire varient de $-0,6$ pour cent dans la région Nord à 0,2 pour cent dans 1'Outaouais. Ce nouvel équilibre migratoire des diverses régions du Québec est sans doute à mettre en relation avec le mouvement de "contre-urbanisation" observé au Québec au cours des années 1970 (Termote et Mongeau, 1983).

Cette image d'équilibre spatial n'est plus valable lorsque l'on considère les deux autres groupes linguistiques : le groupe anglophone du Québec a vu ses effectifs diminuer annuellement de 3 pour cent à cause de ses échanges migratoires avec le reste du Canada, alors que le groupe "autre" perdait 0,5 pour cent (comparativement au chiffre de 0,01 pour cent du groupe français). L'hémorragie migratoire du groupe anglais se manifeste pour toutes les régions du Québec, avec des taux annuels de migration nette variant de $-1,6$ pour cent (Outaouais et Cantons de $1^{\prime}$ Est) à $-5,0$ pour cent (Intérieur) et $-6,0$ pour cent (Nord), 1a région de Montréal perdant en moyenne chaque année (entre 1976 et 1981) 3 pour cent (plus de 20 mille personnes) de ses effectifs anglophones, à cause presque uniquement de son déficit avec le reste du Canada.

Les divers taux dont il a été question jusqu'à présent sont des taux conceptuellement identiques aux taux bruts de natalité et de mortalité, en ce sens qu'il n'est pas tenu compte de la structure par âge. Pour évaluer le niveau de la migration en éliminant les effets de la structure par âge, on peut calculer, par analogie avec le taux brut de reproduction, ce que l'on a appelé le taux brut de migraproduction (obtenu en sommant les taux de migration par âge et en multipliant le résultat par cinq, le nombre d'années de chaque groupe d'âge).

Ces taux de migraproduction (taux bruts car ils ne tiennent pas compte de la mortalité) confirment pour l'essentiel les résultats obtenus à partir des taux de migration, à savoir que les francophones du Québec sont - et de loin - les moins mobiles, surtout ceux de Montréal, dont le niveau de la courbe d'émigration est près de la moitié de celui des francophones des autres régions (sauf celle de l'Intérieur). Ce sont cependant les membres du groupe "autre" de Montréal qui ont manifesté le niveau d'émigration le plus bas $(0,68$ au 1 ieu de 0,78 pour les francophones). Les anglophones de Montréal ont un niveau d'émigration environ quatre à cinq fois plus élevé que celui des deux autres groupes. Lorsque cependant on ne considère que les seules relations entre $1^{\prime}$ ensemble du Québec et le reste du Canada (chiffres non présentés dans le tableau 4), on obtient des écarts entre groupes linguistiques encore plus élevés que ceux obtenus à partir des taux d'émigration totale. Au lieu d'avoir des rapports de 1 à 4 à 23 pour 
respectivement les groupes français, autre et anglais, on obtient des rapports de 1 à 5 à 26 . En d'autres termes, l'élimination des différences dans la structure par âge a pour effet d'accroître encore davantage les disparités linguistiques dans la propension à l'émigration vers le reste du Canada.

Quelle que soit la région d'émigration considérée, 1'âge moyen des émigrants francophones est toujours plus bas que celui des émigrants anglophones, lui-même inférieur à celui des émigrants du groupe "autre". Si $1^{\prime}$ on ne considère que les émigrants du Québec vers le reste du Canada, on peut évaluer l'âge moyen des émigrants au moment de la migration à 23 ans pour les francophones, 27 ans pour les anglophones et 36 ans pour ceux du groupe "autre". Par contre, les immigrants ont un âge moyen nettement plus bas : 19 ans pour les francophones (cela est dû en grande partie au nombre élevé d'enfants en bas âge ayant immigré dans la région de 1'Outaouais), 20 ans pour les anglophones et 24 ans pour les allophones. Le fait que les migrants anglophones aient un âge moyen plus élevé que les migrants francophones doit bien sûr être relié à la structure par âge plus vieille de la population de langue anglaise. En ce qui concerne les migrants allophones, leur âge moyen élevé s'explique sans doute essentiellement par les transferts linguistiques, les enfants n'ayant pas nécessairement gardé la langue d'usage de leurs parents.

En d'autres termes, entre 1976 et 1981, le Québec a sans doute perdu beaucoup d'anglophones dans ses échanges migratoires avec le reste du Canada, mais le peu qu'il a gagné (38 mille personnes) est en moyenne nettement plus jeune (20 ans contre 27 ans) que ce qu'il a perdu (169 mille personnes). Les échanges ont été, comme nous l'avons vu, quasiment équilibrés en ce qui concerne le groupe français, mais encore une fois, le Québec a attiré une population plus jeune (19 ans contre 23 ans) que celle qu'il a perdue. Enfin, si le Québec a perdu onze mille membres du groupe "autre", ces derniers étaient en moyenne beaucoup plus âgés (36 ans contre 24 ans) que les deux milles allophones qu'il a pu attirer. En outre, comme précédemment, le fait de tenir compte des différences dans la structure par âge des populations concernées, accroît les disparités linguistiques : alors que 1 'écart maximum dans $1^{\prime}$ âge moyen observé est de 12 ans pour le flux d'émigration du Québec, il est de 15 ans lorsqu'on considère I'âge moyen standardisé; pour le flux d'immigration, l'écart passe de 5 ans à 8 ans. D'une façon générale, ces observations pour l'ensemble du Québec sont proches de celles que $I^{\prime}$ on peut faire pour chacune de ses régions.

\section{MIGRATION ET MORTALITE : LA TABLE MULTIRÉGIONALE DE MORTALITE}

La caractéristique essentielle de l'approche multirégionale est de permettre une analyse simultanée des divers phénomènes démographiques (Rogers, 1975; Willekens et Rogers, 1978). Nous considérons d'abord la conjonction entre migration et mortalité, c'est-à-dire la table multirégionale de mortalité. 
Tout comme dans la table traditionnelle (non spatiale), on suppose que, au fur et à mesure qu'un individu passe à travers les divers âges de la vie, il est soumis aux conditions de mortalité et de migration observées aujourd'hui (en 1'occurrence, en 1976-1981) pour chacun de ces âges. En outre, puisque nous raisonnons dans un système multirégional, il nous faut faire des hypothèses quant au régime régional de migration et de mortalité à appliquer aux migrants. Nous supposerons qu'un migrant est soumis aux conditions démographiques de la région d'immigration. Sans doute, une telle hypothèse est-elle discutable. $\AA$ cet égard, il importe cependant de souligner que $l^{\prime}$ objectif $n$ 'est pas de réaliser des prévisions quant au comportement futur d'une population: tout comme dans 1'approche traditionnelle, il s'agit de résumer la situation actuelle, plus précisément de voir les implications futures du comportement présent.

Il n'est évidemment pas possible de présenter ici l'ensemble de la table multirégionale de mortalité d'un système comprenant sept régions. Seuls quelques résultats jugés particulièrement significatifs seront discutés. Une première façon de combiner migration et mortalité est de calculer la probabilité de survivre dans la région de naissance. Le tableau 5 présente ces probabilités pour trois âges, que 1 'on peut considérer comme délimitant des étapes importantes dans le cycle de vie d'un individu : 20 ans (entrée sur le marché du travail), 35 ans (mobilité professionnelle et géographique à mi-carrière), et 65 ans (retraite).

On peut ainsi constater que, selon le régime de migration et de mortalité observé entre 1976 et 1981, aucune de nos six régions du Québec ne garderait la moitié de ses nouveau-nés du groupe anglais jusqu'à ce que ces derniers aient atteint leur vingtième année. En d'autres termes, plus de la moitié de ces nouveau-nés anglophones ne contribueront pas à la "force de travaill de leur région de naissance. Au total, 55 pour cent de ces nouveau-nés auraient quitté le Québec pour une autre province. Entre 20 et 35 ans, âges où la mobilité spatiale est la plus forte, la grande majorité de ces "survivants" partiraient à leur tour, de sorte qu'il n'en resterait même pas 20 pour cent. Finalement, seule une infime minorité résiderait dans sa région de naissance tout au long de sa vie active. De toutes les régions, ce sont celles de Montréal et des Cantons de l'Est qui manifestent la capacité de rétention la plus élevée, quoique très faible (à peine 6 ou 7 pour cent des nouveau-nés du groupe anglais de ces régions y seraient encore à 65 ans).

Quant aux nouveau-nés du groupe français, plus du tiers aurait déjà quitté leur région de naissance avant 1 'âge de 20 ans, exception faite pour ceux nés dans les régions de Montréal et de l'Intérieur qui parviendraient à en garder environ les trois quarts. Montréal manifeste d'ailleurs une fois de plus la capacité de rétention la plus élevée : près de la moitié des nouveau-nés du groupe français y serait encore à 1'âge de 65 ans, alors que les autres régions (sauf celle de l'Intérieur) n'en garderaient même pas le quart. 
Tableau 5

Probabilité de survivre dans la région de naissance (en \%), par région et groupe linguistique, 1976-1981

\begin{tabular}{|c|c|c|c|c|}
\hline \multirow{2}{*}{\multicolumn{2}{|c|}{$\begin{array}{l}\text { Région et } \\
\text { groupe } \\
\text { linguistique }\end{array}$}} & \multicolumn{3}{|c|}{$\begin{array}{c}\text { Probabilité de survivre dans la même région } \\
\text { jusqu'à } 1 \text { 'âge de }\end{array}$} \\
\hline & & 20 ans & 35 ans & 65 ans \\
\hline Outaouais & $\begin{array}{l}-F \\
-A \\
-0\end{array}$ & $\begin{array}{l}63 \\
34 \\
56\end{array}$ & $\begin{array}{r}40 \\
9 \\
37\end{array}$ & $\begin{array}{r}23 \\
3 \\
15\end{array}$ \\
\hline Montréal & $\begin{array}{l}-F \\
-A \\
-0\end{array}$ & $\begin{array}{l}77 \\
45 \\
89\end{array}$ & $\begin{array}{l}66 \\
20 \\
76\end{array}$ & $\begin{array}{r}45 \\
6 \\
55\end{array}$ \\
\hline Cantons & $\begin{array}{l}-F \\
-A \\
-0\end{array}$ & $\begin{array}{l}61 \\
43 \\
42\end{array}$ & $\begin{array}{l}38 \\
15 \\
19\end{array}$ & $\begin{array}{r}24 \\
7 \\
8\end{array}$ \\
\hline Intérieur & $\begin{array}{l}-F \\
-A \\
-0\end{array}$ & $\begin{array}{l}72 \\
18 \\
61\end{array}$ & $\begin{array}{r}54 \\
3 \\
35\end{array}$ & $\begin{array}{r}39 \\
1 \\
9\end{array}$ \\
\hline Gaspésie & $\begin{array}{l}-F \\
-A \\
-0\end{array}$ & $\begin{array}{l}60 \\
34 \\
81\end{array}$ & $\begin{array}{r}35 \\
8 \\
60\end{array}$ & $\begin{array}{r}23 \\
3 \\
15\end{array}$ \\
\hline Nord & $\begin{array}{l}-F \\
-A \\
-0\end{array}$ & $\begin{array}{l}64 \\
18 \\
87\end{array}$ & $\begin{array}{r}41 \\
3 \\
75\end{array}$ & $\begin{array}{r}23 \\
0 \\
45\end{array}$ \\
\hline $\begin{array}{l}\text { Canada- } \\
\text { Québec }\end{array}$ & $\begin{array}{l}-F \\
-A \\
-0\end{array}$ & $\begin{array}{l}70 \\
97 \\
97\end{array}$ & $\begin{array}{l}53 \\
95 \\
95\end{array}$ & $\begin{array}{l}38 \\
79 \\
78\end{array}$ \\
\hline Québec & $\begin{array}{l}-F \\
-A \\
-0\end{array}$ & $\begin{array}{l}95 \\
45 \\
89\end{array}$ & $\begin{array}{l}91 \\
19 \\
77\end{array}$ & $\begin{array}{r}71 \\
7 \\
56\end{array}$ \\
\hline
\end{tabular}


C'est cependant auprès du groupe "autre" que la région de Montréal montre la capacité d'absorption la plus forte : près de 90 pour cent des personnes appartenant à ce groupe y seraient encore à 20 ans (contre 77 pour cent pour le groupe français et 45 pour cent pour le groupe anglais), les trois quarts y survivraient jusqu'à 35 ans (contre les deux tiers des personnes du groupe français et un cinquième de celles du groupe anglais), et 55 pour cent y seraient encore à l'âge de leur retraite (contre respectivement 45 pour cent et 6 pour cent pour les deux autres groupes). Toutes les autres régions (sauf la région Nord) manifestent une capacité de rétention moins forte pour les personnes du groupe "autre" que pour celles du groupe français. Il faut également ajouter que si Montréal garde plus facilement les membres du groupe "autre" que ceux du groupe français, il $n^{\prime}$ en va pas de même pour l'ensemble du Québec: c'est la très faible propension des personnes du groupe "autre" de Montréal à émigrer vers une autre région du Québec qui rend compte de la forte capacité de rétention de Montréal pour ce groupe.

La désagrégation de l'espérance de vie selon le lieu de résidence est certes l'un des résultats les plus intéressants de la conjonction entre migration et mortalité. Le tableau 6 présente, pour chacun des trois groupes linguistiques, et pour chacune des régions, le nombre d'années qu'un individu né dans une région peut espérer vivre dans chacune des régions de notre système régional, en supposant que tout au long de sa vie, il est soumis aux conditions de migration et de mortalité observées en 1976-1981.

L'espérance de vie à la naissance d'un nouveau-né du groupe francophone est quasiment la même quelle que soit sa région de naissance au Québec. On remarquera, en comparant avec les résultats du tableau 3, que la migration a pour effet d'uniformiser encore plus l'espérance de vie à la naissance : sans migration, celle-ci varie de 71,2 à 72,9 années, au lieu d'un écart de 72,0 à 72,4 années dans le cas de 1 'espérance de vie avec migration. Cette homogénéisation spatiale de l'espérance de vie n'est guère surprenante : par définition, si on laisse un individu quitter une région de sur- (sous-) mortalité et qu'on lui applique les conditions de mortalité de la région où il immigre, 1 'espérance de vie augmentera pour l'individu né dans la région de surmortalité et diminuera pour celui né dans la région de sous-mortalité.

Cette espérance "multirégionale" de vie à la naissance des francophones est cependant encore toujours plus faible que celle des anglophones et des allophones (sauf pour ces derniers, lorsqu'ils sont nés dans la région Nord ou 1'Outaouais). L'espérance de vie à la naissance varie en effet, selon les régions, de 74,5 à 75,0 années pour un anglophone et de 71,6 à 78,1 années pour un allophone. Encore une fois, on peut observer 1 'effet d'homogénéisation du niveau de 1 'espérance de vie, suite à la migration : l'espérance de vie "sans migration" varie de 74,9 à 79,7 années pour un anglophone, et de 69,5 à 82,3 années pour un allophone (vu les petits effectifs, ces derniers chiffres sont cependant sujets à caution). 
Tableau 6

Espérance de vie à la naissance, selon la région de résidence, 1976-1981 A- Groupe français

\begin{tabular}{|c|c|c|c|c|c|c|c|c|c|}
\hline \multirow{2}{*}{$\begin{array}{l}\text { Région de } \\
\text { naissance }\end{array}$} & \multicolumn{7}{|c|}{ Région de résidence } & \multirow{2}{*}{ Total } & \multirow{2}{*}{$\begin{array}{c}\text { Part } \\
\text { (exportée) } \\
(\text { en \%) }\end{array}$} \\
\hline & 0 & M & $\mathrm{C}$ & I & G & $\mathrm{N}$ & $C-Q$ & & \\
\hline Outaouais (0) & 35,0 & 13,4 & 1,3 & 7,9 & 1,4 & 3,0 & 10,1 & 72,2 & 52 \\
\hline Montréal (M) & 0,8 & 51,0 & 2,1 & 10,7 & 1,7 & 2,5 & 3,3 & 72,2 & 29 \\
\hline Cantons (C) & 0,8 & 17,4 & 34,2 & 12,8 & 1,3 & 2,6 & 3,4 & 72,4 & 53 \\
\hline Intērieur (I) & 0,8 & 15,7 & 2,5 & 45,3 & 1,9 & 3,3 & 2,9 & 72,4 & 38 \\
\hline Gaspésie (G) & 0,7 & 14,9 & 1,7 & 13,0 & 32,9 & 5,4 & 3,6 & 72,3 & 54 \\
\hline Nord $(\mathrm{N})$ & 1,2 & 14,2 & 1,8 & 12,0 & 3,0 & 35,4 & 4,4 & 72,0 & 51 \\
\hline $\begin{array}{l}\text { Canada- } \\
\text { Québec }(C-Q)\end{array}$ & 5,5 & 11,2 & 1,2 & 6,1 & 1,5 & 3,2 & 44,1 & 72,9 & 40 \\
\hline
\end{tabular}

B- Groupe anglais

\begin{tabular}{|c|c|c|c|c|c|c|c|c|c|}
\hline \multirow{2}{*}{$\begin{array}{l}\text { Région de } \\
\text { naissance }\end{array}$} & \multicolumn{7}{|c|}{ Région de résidence } & \multirow{2}{*}{ Total } & \multirow{2}{*}{$\begin{array}{c}\text { Part } \\
\text { *exportée } \\
(\text { en \%) }\end{array}$} \\
\hline & 0 & $\mathbf{M}$ & $\mathrm{C}$ & $I$ & G & $\mathrm{N}$ & $C-Q$ & & \\
\hline Outaouais (0) & 16,2 & 1,3 & 0,1 & 0,1 & 0,1 & 0,1 & 57,0 & 74,8 & 78 \\
\hline Montréal (M) & 0,1 & 22,0 & 0,4 & 0,2 & 0,0 & 0,1 & 52,0 & 74,7 & 71 \\
\hline Cantons (C) & 0,2 & 5,7 & 20,0 & 0,4 & 0,0 & 0,1 & 48,2 & 74,6 & 73 \\
\hline Intérieur (I) & 0,2 & 7,4 & 1,0 & 10,0 & 0,3 & 0,3 & 55,7 & 75,0 & 87 \\
\hline Gaspésie (G) & 0,1 & 5,0 & 0,2 & 0,9 & 15,9 & 0,3 & 52,2 & 74,7 & 79 \\
\hline Nord (N) & 0,4 & 4,5 & 0,4 & 0,8 & 0,2 & 11,1 & 57,0 & 74,5 & 85 \\
\hline $\begin{array}{l}\text { Canada- } \\
\text { Québec }(C-Q)\end{array}$ & 0,1 & 0,4 & 0,0 & 0,0 & 0,0 & 0,0 & 73,4 & 74,1 & 1 \\
\hline \multicolumn{10}{|c|}{ C- Groupe "autre } \\
\hline \multirow{2}{*}{$\begin{array}{l}\text { Région de } \\
\text { naissance }\end{array}$} & \multicolumn{7}{|c|}{ Région de résidence } & \multirow{2}{*}{ Total } & \multirow{2}{*}{$\begin{array}{c}\text { Part } \\
\text { «exportée) } \\
(\text { en \%) }\end{array}$} \\
\hline & 0 & $\mathbf{M}$ & C & $I$ & G & $\mathrm{N}$ & $\mathrm{C}-\mathrm{Q}$ & & \\
\hline Outaouals (0) & 30,5 & 5,0 & 0,6 & 0,2 & 0,0 & 1,7 & 34,2 & 72,2 & 58 \\
\hline Montréal (M) & 0,1 & 60,2 & 0,1 & 0,4 & 0,0 & 0,3 & 16,2 & 77,3 & 22 \\
\hline Cantons (C) & 0,0 & 33,5 & 21,2 & 1,7 & 0,0 & 0,7 & 19,1 & 76,3 & 72 \\
\hline Intérieur (I) & 0,0 & 25,0 & 0,3 & 28,5 & 0,0 & 1,6 & 19,9 & 75,3 & 62 \\
\hline Gaspésie (G) & 0,9 & 17,9 & 0,1 & 0,1 & 41,0 & 2,8 & 15,3 & 78,1 & 48 \\
\hline Nord $(N)$ & 0,3 & 7,3 & 0,0 & 0,6 & 0,0 & 54,8 & 8,6 & 71,6 & 24 \\
\hline $\begin{array}{l}\text { Canada- } \\
\text { Québec }(C-Q)\end{array}$ & 0,1 & 0,7 & 0,0 & 0,0 & 0,0 & 0,2 & 73,1 & 74,2 & 1 \\
\hline
\end{tabular}


Comme un anglophone né dans le reste du Canada est très peu porté à séjourner au Québec, son espérance de vie "spatialisée" n'est pas significativement différente de son espérance de vie "sans migration" (74,1 contre 74,2 années), alors qu'un francophone né dans le reste du Canada perd une année d'espérance de vie si on le laisse émigrer au Québec, où les conditions de mortalité sont moins favorables ( 1 'espérance de vie spatiale est de 72,9 années au lieu des 73,8 années de 1'espérance de vie "sans migration").

La prise en compte simultanée de la migration et de la mortalité implique qu'un certain nombre d'années "à vivre" seront en fait vécues en dehors de la région de naissance ou de la région de résidence actuelle (pas nécessairement de façon successive, à cause des migrations multiples). Le pourcentage du nombre d'années qu'un nouveau-né peut s'attendre à vivre en dehors de sa région de naissance est un bon indicateur de la capacité de rétention de cette dernière. Ce pourcentage est présenté dans la dernière colonne du tableau 6, sous le vocable "part exportée". I1 démontre, une fois de plus, à quel point la région de Montréal domine le système régional québécois (tel que nous 1 'avons défini). Quel que soit le groupe linguistique considéré, c'est Montréal qui perd le moins d'"années à vivrell : 22 pour cent de 1'espérance totale de vie d'un nouveau-né du groupe "autre", 29 pour cent de celle d'un nouveau-né du groupe français et 71 pour cent de celle d'un nouveau-né du groupe anglais. Un nouveau-né du groupe français né en dehors de Montréal peut s'attendre à vivre plus de la moitié de sa vie en dehors de sa région de naissance (sauf s'il est né dans la région de l'Intérieur).

Non seulement la région de Montréal perd-elle relativement moins d' "années à vivrel" par un nouveau-né francophone mais encore reçoit-elle l'essentiel des années perdues par les autres régions : un individu né en dehors de Montréal peut espérer vivre à Montréal entre 13 années (s'il est né dans l'Outaouais) et 17 années ( $s^{\prime} i l$ est né dans les Cantons de 1'Est). Même un francophone né dans le reste du Canada peut s'attendre à vivre 11 années de sa vie à Montréal. Par contre, s'il est né au Québec, ce francophone ne passera que peu d'années ( 3 à 4 ans) en dehors du Québec, sauf s'il est né dans l'Outaouais. Mais un anglophone né au Québec passerait environ les deux tiers de sa vie en dehors du Québec, soit entre 48 années ( $s^{\prime} i l$ est né dans les Cantons de $l^{\prime}$ Est) et 57 années ( $s^{\prime} i l$ est né dans 1 'Outaouais ou la région Nord); $i 1$ ne vivrait que de 10 à 22 ans dans sa région de naissance au Québec, et quasiment pas dans une région québécoise autre que celle de sa naissance.

Le Québec fait donc clairement partie de l'uespace de viell des francophones nés au Canada en dehors de la province : ces derniers peuvent s'attendre à vivre environ 40 pour cent de leur vie au Québec. Par contre, un nouveau-né du groupe anglais ou du groupe "autre" du reste du Canada ne séjournera quasiment jamais au Québec. 
Les implications socio-économiques (et politiques) de ces transferts interrégionaux d'"années à vivre", caractérisés par la dominance de la région de Montréal au sein du système régional québécois et par 1'absence d'intégration du Québec dans 1'espace de vie des anglophones et allophones du reste du Canada, ne peuvent évidemment pas être sous-estimées. I1 ne faut pas oublier qu'une grande partie de ces transferts d' "années à vivre" concernent les étapes du cycle de vie qui économiquement et démographiquement sont les plus "productives". Certaines régions doivent "supporter" pendant une vingtaine d'années des individus qui ne contribueront que fort peu à la force de production et de reproduction de leur région de naissance. Ce n'est pas le lieu ici d'analyser les implications socio-économiques de ce phénomène. Nous pouvons cependant, grâce à notre approche multirégionale, en évaluer ici une implication démographique, à savoir celle qui concerne le renouvellement de l.a population.

\section{MIGRATION, FÉCONDITÉ ET MORTALITE : LE TAUX SPATIAL DE REPRODUCTION NETTE}

Tout comme 1'espérance de vie peut être désagrégée selon la région de résidence où seront vécues ces "années à vivre", de même le taux de reproduction nette peut être désagrégé selon la région où résideront les parents au moment de la naissance de leur descendance "attendue" (région que $l^{\prime}$ on supposera ici identique à la région de naissance de cette descendance). Le tableau 7 présente, pour chacun des groupes linguistiques et pour chacune des régions de naissance des parents, le taux spatial de reproduction nette, obtenu en combinant les taux de migration, de fécondité et de mortalité observés en 1976-1981.

Comme dans le cas de l'espérance de vie, la migration a pour effet de réduire les disparités régionales dans le taux de reproduction nette : alors que le taux de reproduction nette sans migration varie, au Québec, de 0,78 à 1,01 pour les francophones, de 0,62 à 0,99 pour les anglophones, et de 0,96 à 1,70 pour les allophones, ces mêmes taux, calculés avec migration, varient respectivement de 0,81 à 0,94 , de 0,76 à 0,83 , et de 0,97 à 1,62 . Cette homogénéisation spatiale est bien sûr liée à $1^{\prime}$ hypothèse selon laquelle les migrants adoptent le régime de fécondité de leur région d'immigration.

La migration a ainsi nécessairement pour effet de réduire le taux de reproduction nette des régions à haute fécondité, et d'augmenter celui des régions à faible fécondité. On peut donc constater, par exemple, que sans migration et en négligeant la mortalité, les francophones de la région Nord sont les seuls de ce groupe linguistique à assurer - de justesse - le renouvellement de leur population (le taux brut de reproduction y est de 1,05). La prise en compte de la mortalité ne modifie pas cette constatation (taux net, sans migration, de 1,01). Mais lorsqu'on intègre également 1a migration, il en résulte que la population francophone née dans la région Nord n'assure plus son propre renouvellement (le taux spatial de 
Tableau 7

Taux spatial de reproduction nette, par région et groupe 11nguistique, 1976-1981

A- Groupe francals

\begin{tabular}{|c|c|c|c|c|c|c|c|c|c|c|c|}
\hline \multirow{2}{*}{$\begin{array}{l}\text { Régtion de } \\
\text { nalssance } \\
\text { des parents }\end{array}$} & \multicolumn{7}{|c|}{ Région de naissance de la descendance } & \multirow[t]{2}{*}{ Total } & \multirow{2}{*}{$\begin{array}{c}\text { Part } \\
\text { (exportée } \\
(\text { en } \%)\end{array}$} & \multicolumn{2}{|c|}{$\begin{array}{l}\text { Taux sans } \\
\text { migration }\end{array}$} \\
\hline & 0 & M & $\mathrm{C}$ & I & G & $\mathbf{N}$ & $C-Q$ & & & Brut & Net \\
\hline Outaouais (0) & 0,46 & 0,14 & 0,01 & 0,08 & 0,01 & 0,04 & 0,12 & 0,86 & 47 & 0,87 & 0,84 \\
\hline Montréal (M) & 0,01 & 0,58 & 0,02 & 0,11 & 0,02 & 0,03 & 0,04 & 0,81 & 28 & 0,80 & 0,78 \\
\hline Cantons (C) & 0,01 & 0,18 & 0,47 & 0,14 & 0,01 & 0,03 & 0,04 & 0,89 & 47 & 0,95 & 0,92 \\
\hline Intérleur (I) & 0,01 & 0,17 & 0,03 & 0,55 & 0,02 & 0,05 & 0,03 & 0,85 & 36 & 0,89 & 0,86 \\
\hline Gaspésie (G) & 0,01 & 0,16 & 0,02 & 0,15 & 0,43 & 0,08 & 0,04 & 0,88 & 52 & 0,95 & 0,92 \\
\hline Nord $(N)$ & 0,01 & 0,14 & 0,02 & 0,13 & 0,03 & 0,55 & 0,05 & 0,94 & 42 & 1,05 & 1,01 \\
\hline $\begin{array}{l}\text { Canada- } \\
\text { Québec }(C-0))\end{array}$ & 0,07 & 0,11 & 0,01 & 0,06 & 0,02 & 0,04 & 0,62 & 0,92 & 33 & 0,98 & 0,95 \\
\hline
\end{tabular}

B- Groupe anglals

\begin{tabular}{|c|c|c|c|c|c|c|c|c|c|c|c|}
\hline \multirow{2}{*}{$\begin{array}{l}\text { Région de } \\
\text { naissance } \\
\text { des parents }\end{array}$} & \multicolumn{7}{|c|}{ Région de naissance de la descendance } & \multirow{2}{*}{ Total } & \multirow{2}{*}{$\begin{array}{c}\text { Part } \\
\text { "exportée" } \\
(\text { en } \%)\end{array}$} & \multicolumn{2}{|c|}{$\begin{array}{l}\text { Taux sans } \\
\text { migration }\end{array}$} \\
\hline & 0 & $\mathbf{M}$ & C & I & $\mathbf{G}$ & $\mathrm{N}$ & $C-Q$ & & & Brut & Net \\
\hline Outaouais (0) & 0,16 & 0,01 & 0,00 & 0,00 & 0,00 & 0,00 & 0,66 & 0,83 & 81 & 0,77 & 0,75 \\
\hline Montréal (M) & 0,01 & 0,20 & 0,00 & 0,00 & 0,00 & 0,00 & 0,55 & 0,76 & 74 & 0,63 & 0,62 \\
\hline Cantons (C) & 0,00 & 0,07 & 0,21 & 0,00 & 0,00 & 0,00 & 0,52 & 0,80 & 73 & 0,82 & 0,80 \\
\hline Intérieur (I) & 0,00 & 0,09 & 0,01 & 0,06 & 0,00 & 0,01 & 0,62 & 0,80 & 92 & 0,75 & 0,75 \\
\hline Gaspésie（G） & 0,00 & 0,06 & 0,00 & 0,01 & 0,17 & 0,01 & 0,58 & 0,83 & 80 & 0,94 & 0,92 \\
\hline Nord (N) & 0,00 & 0,06 & 0,01 & 0,01 & 0,00 & 0,10 & 0,66 & 0,83 & 89 & 1,01 & 0,99 \\
\hline $\begin{array}{l}\text { Canada- } \\
\text { Québec }(C-Q)\end{array}$ & 0,00 & 0,00 & 0,00 & 0,00 & 0,00 & 0,00 & 0,84 & 0,85 & 1 & 0,87 & 0,85 \\
\hline
\end{tabular}

\section{C- Groupe "autre"}

\begin{tabular}{|c|c|c|c|c|c|c|c|c|c|c|c|}
\hline \multirow{2}{*}{$\begin{array}{l}\text { Région de } \\
\text { natssance } \\
\text { des parents }\end{array}$} & \multicolumn{7}{|c|}{ Réglon de natssance de la descendance } & \multirow{2}{*}{ Total } & \multirow{2}{*}{$\begin{array}{c}\text { Part } \\
\text { exportée } \\
(\text { en } \%)\end{array}$} & \multicolumn{2}{|c|}{$\begin{array}{l}\text { Taux sans } \\
\text { migration }\end{array}$} \\
\hline & 0 & $\mathbf{M}$ & C & I & G & $\mathrm{N}$ & $C-Q$ & & & Brut & Net \\
\hline Outaouals (0) & 0,62 & 0,04 & 0,01 & 0,00 & 0,00 & 0,05 & 0,38 & 1,12 & 44 & 1,28 & 1,20 \\
\hline Montréal (M) & 0,01 & 0,82 & 0,00 & 0,01 & 0,00 & 0,01 & 0,13 & 0,97 & 15 & 0,98 & 0,96 \\
\hline Cantons (C) & 0,00 & 0,45 & 0,41 & 0,04 & 0,00 & 0,01 & 0,16 & 1,07 & 62 & 1,28 & 1,24 \\
\hline Intērieur (I) & 0,00 & 0,27 & 0,00 & 0,61 & 0,00 & 0,04 & 0,16 & 1,09 & 44 & 1,22 & 1,17 \\
\hline Gaspés1e（G） & 0,02 & 0,20 & 0,00 & 0,00 & 0,97 & 0,10 & 0,06 & 1,35 & 28 & 1,48 & 1,48 \\
\hline Nord (N) & 0,01 & 0,05 & 0,00 & 0,01 & 0,00 & 1,50 & 0,05 & 1,62 & 7 & 1,83 & 1,70 \\
\hline $\begin{array}{l}\text { Canada- } \\
\text { Québec }(C-Q)\end{array}$ & 0,00 & 0,01 & 0,00 & 0,00 & 0,00 & 0,01 & 0,97 & 0,99 & 2 & 1,02 & 0,99 \\
\hline
\end{tabular}


reproduction nette baisse à 0,94). Par contre, les anglophones de la région de Montréal, dont le taux sans migration est le plus bas de tous $(0,63$ ou 0,62 selon que $l^{\prime}$ on néglige ou non la mortalité), voient augmenter significativement (jusqu'à 0,76 ) leur taux de reproduction nette lorsqu'on tient compte de la migration. Comme ces anglophones vont surtout séjourner dans le reste du Canada où ils sont soumis à un régime de fécondité plus élevé, leur taux de reproduction s'élève.

Suite à la migration, le "renouvellement" d'une population ne se fera pas nécessairement dans la région de naissance de cette population. En fait, 1 'examen de la diagonale principale de chacun des tableaux 7-A, 7-B et 7-C, montre qu'aucun groupe linguistique dans aucune région ne peut espérer, étant donné les conditions de fécondité, de migration et de mortalité observées entre 1976 et 1981, renouveler sa population en faisant appel à la seule population du même groupe linguistique née dans la région. En effet, chaque élément de chacune de ces diagonales est toujours inférieur à l'unité (la seule exception concerne la population du groupe "autre" de la région Nord).

En ce qui concerne plus particulièrement le groupe français du Québec, on peut observer qu'entre 28 pour cent (Montréal) et 52 pour cent (Gaspésie) de la descendance attendue naîtrait en dehors de la région de naissance des parents. Dans la plupart des cas cependant, la région de naissance de cette descendance attendue se situe au Québec même (à l'exception de l'Outaouais, pour lequel un septième de la descendance est attendue en dehors du Québec).

Dans le cas du groupe anglais du Québec, environ les trois quarts de la descendance est attendue en dehors du Québec (les deux tiers dans le cas des Cantons de 1'Est). Le groupe de langue anglaise a donc non seulement un taux de reproduction très faible, mais en outre on peut s'attendre à ce que la plupart des rares enfants des natifs québécois de ce groupe linguistique naissent en dehors du Québec. Dans les conditions de fécondité, de migration et de mortalité observées entre 1976 et 1981, le groupe anglais ne peut certes pas compter sur sa seule population née dans une région pour se renouveler dans cette région : le taux "local" de reproduction nette varie de 0,06 à 0,21 ! De plus, l'apport d'une descendance anglophone venue d'une autre région du Québec est quasiment nul, vu la très faible propension des anglophones à migrer entre les régions du Québec.

A cet égard, le groupe "autre" se trouve dans une bien meilleure situation: une fécondité relativement élevée, jointe à une faible émigration, permet à cette population d'atteindre un taux local de 0,82 à Montréal (comparé à un taux de 0,58 pour le groupe français et de 0,20 pour le groupe anglais). Il est évident, cependant, que la prise en compte de la mobilité linguistique ferait baisser significativement les taux "locaux" du groupe "autre". 
Le groupe anglais (pas plus d'ailleurs que le groupe "autrel) ne peut guère compter sur une descendance "importée" du reste du Canada : en moyenne, à peine un pour cent des enfants attendus d'un anglophone de cette région naîtrait au Québec. Même en pondérant pour tenir compte des effectifs en cause, il est clair que le "déficit reproductif potentiel" du groupe anglais du Québec, résultant de l'action combinée d'une très faible fécondité et d'une très forte émigration des membres de ce groupe, ne pourra pas être compensé - et de loin - par un apport du groupe anglais du reste du Canada, du moins sous le régime démographique observé au cours des années 1976-1981.

\section{CONCLUSION}

Qu'il y ait des différences considérables dans le niveau de fécondité, de migration et de mortalité de chacun des groupes linguistiques au Québec, n'est certes pas nouveau. Cependant, lorsque ces phénomènes démographiques, au lieu d'être analysés séparément, sont considérés simultanément, selon ce qu'il est convenu d'appeler une perspective "multirégionale", nombre de conclusions que $1^{\prime}$ on avait pu dégager précédemment doivent être nuancées. Deux apports particulièrement significatifs de cette analyse multirégionale nous semblent dignes d'être soulignés.

Ainsi, nous avons pu constater que lorsqu'on intègre la migration à l'analyse de la fécondité et de la mortalité, les disparités régionales en matière de fécondité et de mortalité sont nettement plus faibles (du moins si 1 'on accepte que les migrants adoptent le régime démographique de la région d'immigration).

Par contre, si la migration a pour effet de réduire les disparités régionales de fécondité et de mortalité de chacun des groupes linguistiques, les disparités linguistiques en ce qui concerne l'impact démographique de cette migration pour une région donnée sont apparues bien plus importantes que ce que $1^{\prime}$ on pouvait supposer. En effet, selon le régime démographique observé entre 1976 et 1981, le groupe anglophone de chacune des régions du Québec doit s'attendre non seulement à ne pas renouveler ses effectifs, mais à perdre, au profit du reste du Canada, la très grande part des "lannées à vivrell et des "enfants à naître" de ses (rares) nouveau-nés d'aujourd'hui. En ce qui concerne les groupes français et "autrel du Québec, cet impact futur du comportement migratoire présent est relativement faible.

En d'autres termes, pour le Québec, et sur la base du régime démographique de la période 1976-1981, la migration réduit sans doute les disparités régionales dans le comportement de fécondité et de mortalité, mais elle amplifie l'impact démographique des disparités linguistiques de ce comportement. 
ANNEXE

Découpage géographique : divisions de recensement comprises dans chacune des régions du Québec

\begin{tabular}{|c|c|}
\hline REGION & DIVISIONS DE RECENSEMENT \\
\hline Outaouais & Gatineau, Hu11, Papineau, Pontiac \\
\hline Ensemble de Montréal & $\begin{array}{l}\text { Argenteuil, Beauharnois, Chambly, Châteauguay, } \\
\text { Deux-Montagnes, Huntingdon, Iberville, Ile de } \\
\text { Montréal, Ile Jésus, Laprairie, L'Assomption, } \\
\text { Napierville, Rouville, Saint-Jean, Soulanges, } \\
\text { Terrebonne, Vaudreuil, Verchères. }\end{array}$ \\
\hline Cantons de 1 'Est & $\begin{array}{l}\text { Brome, Compton, Missisquoi, Richmond, Shefford, } \\
\text { Sherbrooke, Stanstead. }\end{array}$ \\
\hline Intérieur & $\begin{array}{l}\text { Arthabaska, Bagot, Beauce, Bellechasse, Ber- } \\
\text { thier, Champlain, Dorchester, Drummond, Fronte- } \\
\text { nac, Joliette, Labelle, Lévis, Lotbinière, } \\
\text { Maskinongé, Mégantic, Montcalm, Montmagny, } \\
\text { Montmorency no 1, Montmorency no 2, Nicolet, } \\
\text { Portneuf, Québec, Richelieu, Saint-Hyacinthe, } \\
\text { Saint-Maurice, Wolfe, Yamaska. }\end{array}$ \\
\hline Gaspésie & $\begin{array}{l}\text { Bonaventure, Gaspé-Est, Gaspé-Ouest, Iles-de- } \\
\text { la-Madeleine, Kamouraska, L'Islet, Matane, } \\
\text { Matapédia, Rimouski, Rivière-du-Loup, Témis- } \\
\text { couata. }\end{array}$ \\
\hline Nord & $\begin{array}{l}\text { Abitibi, Charlevoix-Est, Charlevoix-Ouest, } \\
\text { Chicoutimi, Lac-Saint-Jean-Est, Lac-Saint-Jean- } \\
\text { Ouest, Saguenay, Témiscamingue, Territoire du } \\
\text { Nouveau-Québec. }\end{array}$ \\
\hline
\end{tabular}


REFERENCES BIBLIOGRAPHIQUES

ALBERT, Luc, et Brian HARRISON, 1984. "Les données linguistiques des recensements récents au Canadal. Communication présentée au 52ième congrès de 1'ACFAS, section démographie, Québec, 10-11 mai 1984.

LACHAPELLE, Réjean, et Jacques HENRIPIN, 1980. La situation démolinguistique au Canada : évolution passée et prospective. Montréal, Institut de recherches politiques, $391 \mathrm{p}$.

ROGERS, Andrei, 1975. Introduction to Multiregional Mathematical Demography. New York, John Wiley and Sons, 203 p.

TERMOTE, Marc, et Jaë1 MONGEAU, 1983. "L'ampleur de la contre-urbanisation au Québec". Actes du colloque sur "L'exode urbain, ses causes, ses implications et son avenir". Montréal, Université de Montréal, Département de géographie, Yves Brunet éd., 77-88.

TREMBLAY, Marc, 1983. Analyse de la mortalité et de la fécondité selon le groupe 1inguistique, Québec, 1976-1981. Mémoire présenté à 1a Faculté des études supérieures en vue de l'obtention de la mâ̂trise en démographie. Montréal, Université de Montréal, 285 p.

WILLEKENS, Frans, et Andrei ROGERS, 1978. Spatial Population Analysis : Methods and Computer Programs. Laxenburg (Autriche), International Institute for Applied Systems Analysis, 302 p. 


\author{
RÉSUMÉ - SUMMARY - RESUMEN
}

TERMOTE, Marc et Danielle GAUVREAU - LE COMPORTEMENT DEMOGRAPHIQUE DES GROUPES LINGUISTIQUES AU QUÉBEC PENDANT LA PERIODE 1976-1981. UNE ANALYSE MULTIREGIONALE

L'objectif de cet article est d'analyser de façon simultanée, selon ce qu'il est convenu d'appeler l'approche multirégionale, les interactions entre le comportement de fécondité, de migration et de mortalité de chacun des principaux groupes linguistiques du Québec. Les résultats de cette analyse montrent que, sur la base du régime démographique observé au cours de la période 1976-1981, la migration a sans doute pour effet de réduire les disparités régionales dans le comportement de fécondité et de mortalité, majis que par contre elle amplifie considérablement l'impact démographique des disparités linguistiques de ce comportement.

TERMOTE, Marc and Danie11e GAUVREAU - THE DEMOGRAPHIC BEHAVIOR OF LINGUISTIC GROUPS IN QUEBEC DURING THE 1976-1981 PERIOD. A MULTIREGIONAL ANALYSIS.

The purpose of this paper is to analyse simultaneously, according to what is known as the multiregional approach, the interactions between fertility, migration and mortality, for each of the main linguistic groups of Quebec. Results show that, on the basis of the demographic regime observed between 1976 and 1981, while migration probably has reduced regional disparities in fertility and mortality behavior, it also has markedly amplified the demographic impact of linguistic disparities in this behavior.

TERMOTE, Marc y Danielle GAUVREAU - EL COMPORTAMIENTO DEMOGRAFICO DE LOS GRUPOS LINGUISTICOS EN QUEBEC DURANTE EL PERIODO 1976-1981. UN ANALISIS MULTIREGIONAL

E1 objetivo de este artículo es analisar simultáneamente, según el enfoque conocido como multiregional, las interacciones entre 1.a fecundidad, la migración y la mortalidad de cada uno de los grupos lingüisticos de Quebec. Los resultados muestran que, en base al régimen demogrảifico observado durante el período 1976-1981, la migración ha probablemente contribuido a disminuir las disparidades regionales en lo que respecta a la fecundidad y a la mortalidad, pero por otro lado, ésta ha aumentado considerablemente el impacto demográfico de las disparidades lingüísticas en este comportamiento. 